\title{
One-leg rise performance and associated knee kinematics in ACL-deficient and ACL- reconstructed persons 23 years post-injury
}

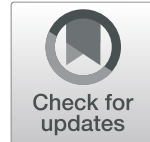

\author{
Andrew Strong $^{1 *}$ DD, Eva Tengman ${ }^{1}$, Divya Srinivasan ${ }^{2}$ and Charlotte K. Häger ${ }^{1}$
}

\begin{abstract}
Background: Research indicates reduced knee function and stability decades after anterior cruciate ligament (ACL) injury. Assessment requires reliable functional tests that discriminate such outcomes from asymptomatic knees, while providing suitable loading for different populations. The One-leg rise (OLR) test is common in clinics and research but lacks scientific evidence for its implementation. Our cross-sectional study compared performance including knee kinematics of the OLR between ACL-injured persons in the very long term to controls and between legs within these groups, and assessed the within-session reliability of the kinematics.
\end{abstract}

Methods: Seventy ACL-injured individuals (mean age $46.9 \pm 5.4$ years) treated with either reconstructive surgery and physiotherapy $\left(\mathrm{ACL}_{R} ; n=33\right.$ ) or physiotherapy alone ( $\left.A C L_{P T} ; n=37\right)$, on average 23 years post-injury, and 33 age- and sex-matched controls (CTRL) attempted the OLR. Participants completed as many repetitions as possible to a maximum of 50 while recorded by motion capture. We compared between all groups and between legs within groups for total repetitions and decomposed the OLR into movement phases to compare phase completion times, maximum and range of knee abduction and adduction angles, and mediolateral knee control in up to 10 repetitions per participant.

Results: ACLPT performed significantly fewer OLR repetitions with their injured leg compared to the CTRL non-dominant leg (medians 15 and 32, respectively) and showed significantly greater knee abduction than $A C L_{R}$ and $C T R L$ (average $2.56^{\circ}-3.69^{\circ}$ depending on phase and leg). Distribution of repetitions differed between groups, revealing $59 \%$ of ACLpT unable to complete more than 20 repetitions on their injured leg compared to $33 \% \mathrm{ACL}_{R}$ and $36 \% \mathrm{CTRL}$ for their injured and non-dominant leg, respectively. Within-session reliability of all kinematic variables for all groups and legs was high (ICC 3,10 0.97-1.00, 95\% Cl 0.95-1.00, SEM 0.93-1.95\%).

Conclusions: Negative outcomes of OLR performance, particularly among ACLPT, confirm the need to address aberrant knee function and stability even decades post-ACL injury. Knee kinematics derived from the OLR were reliable for asymptomatic and ACL-injured knees. Development of the OLR protocol and analysis methods may improve its discriminative ability in identifying reduced knee function and stability among a range of clinical populations.

Keywords: Knee injury, Knee control, Knee function, Lower limb, Osteoarthritis, Clinical assessment, Motion analysis, Biomechanics

\footnotetext{
* Correspondence: andrew.strong@umu.se

${ }^{1}$ Department of Community Medicine and Rehabilitation, Physiotherapy,

Umeå University, SE-90187 Umeå, Sweden

Full list of author information is available at the end of the article
}

(c) The Author(s). 2019 Open Access This article is distributed under the terms of the Creative Commons Attribution 4.0 International License (http://creativecommons.org/licenses/by/4.0/), which permits unrestricted use, distribution, and reproduction in any medium, provided you give appropriate credit to the original author(s) and the source, provide a link to the Creative Commons license, and indicate if changes were made. The Creative Commons Public Domain Dedication waiver (http://creativecommons.org/publicdomain/zero/1.0/) applies to the data made available in this article, unless otherwise stated. 


\section{Background}

Anterior cruciate ligament (ACL) injury often negatively affects knee function not only in the short term but also in the long term despite rehabilitative interventions [1]. Treatment involves either physiotherapy alone or in conjunction with additional reconstructive surgery, with conflicting evidence as to the best approach and a lack of informed guidance for individual tailoring. Regardless of treatment strategy, there is an elevated risk for reinjury/secondary injury during the subsequent years [2], as well as for longer term problems such as knee osteoarthritis (OA) [1]. Studies investigating the very longterm (> 20 years) effects of ACL injury on knee function are, however, scarce.

Clinically evaluating knee function following ACL injury often includes, for example, thigh muscle strength, knee range of motion, and jumping/hopping ability. The injured leg is commonly compared to the contralateral non-injured leg using the Limb Symmetry Index (LSI), where achieving $>90 \%$ of the outcome measures is a return-to-sport criteria [3]. However, the LSI has been shown to overestimate post-ACL injury quadriceps strength and hop ability $[4,5]$. To complement the LSI, assessing movement quality during functional tests may reveal movement patterns that potentially predispose this group to further knee-related issues [6]. Reliable functional tests are therefore needed that can discriminate such patterns from asymptomatic knees while providing suitable loading for different populations, including those of an older age for longer term assessment.

The One-leg rise (OLR) test, involving standing and sitting from a stool with only 1 foot on the ground, has been applied in clinics and research to assess knee function. Thorstensson et al., (2004) found that chronic knee pain sufferers unable to perform 20 repetitions of the OLR were more likely to develop radiographic knee OA 5 years later [7]. The OLR was also more sensitive than gait at identifying changes in peak adduction moment following an exercise programme among the same cohort [8]. A one-leg test may be particularly advantageous when assessing ACL-injured individuals, who have been shown to reduce loading of the injured leg during double-leg squats [9]. In fact, worse knee confidence on average 9 years post-ACL reconstruction has been shown to be associated with poorer performance of the OLR [10]. Moreover, at 510 years post-ACL reconstruction, worse performance of the OLR has also been associated with greater tibiofemoral OA severity [11]. The OLR may thus be a relevant test of lower limb function among ACLinjured persons where both performance regarding the number of repetitions achieved and knee kinematics are of interest.
The potential added value of knee kinematics during the OLR would facilitate assessments of knee joint stability, defined here in accordance with Riemann and Lephart [12] as the ability to remain or promptly return to proper alignment, something that is believed to be a major contributing factor to long-term post-ACL injury knee problems such as OA [13]. Indeed, greater knee abduction of the injured leg compared to the non-injured leg during a one-leg half squat has been seen among non-operated ACL-injured males and females [14]. Greater knee abduction was also observed for the injured leg of non-operated ACL-injured persons compared to controls during tests such as a mini-squat, one-leg half squat and rising from half-kneeling [15]. Additionally, mediolateral knee control, as assessed by measures of knee position in the frontal plane, has been shown to be worse among ACL-injured persons compared to controls during a one-leg hop for distance [16]. Among ACL-injured males, poorer mediolateral knee control during a drop jump was associated with worse knee proprioception [17]. Thus, measures of mediolateral knee control during the OLR may provide additional valuable information regarding knee function among ACL-injured persons. However, a necessary first step before studying OLR knee kinematics to interpret knee function, is to assess the within-session reliability firstly among individuals with asymptomatic knees and secondly among the population of interest, something which we believe has not been done before.

Our aims in this study were to 1) assess the discriminative ability of OLR performance and knee kinematic outcome measures among ACL-injured persons, treated with and without surgical reconstruction, in the very long term after injury between the injured and non-injured legs and to controls without knee complaints, and 2) assess the within-session reliability of knee kinematics during execution of the OLR among asymptomatic individuals and ACL-injured cohorts. We hypothesised that both ACLinjured groups would show worse knee function and stability of the injured leg compared to the non-dominant leg of controls and to their non-injured contralateral leg, as characterised by significantly fewer OLR repetitions and greater knee abduction/adduction range of motion. We further hypothesised that the knee kinematics would show high within-session reliability.

\section{Methods \\ Participants}

This study forms part of the KACL20-study (Knee injury Anterior Cruciate Ligament after more than 20 years), a cross-sectional research programme involving two ACL cohorts and a control group: 1) 33 ACL-injured persons treated with reconstructive surgery and physiotherapy $\left.\left(\mathrm{ACL}_{\mathrm{R}}\right), 2\right) 37 \mathrm{ACL}$-injured persons treated with physiotherapy only $\left(\mathrm{ACL}_{\mathrm{PT}}\right)$, and 3) 33 age- and sex-matched controls 
(CTRL) with asymptomatic knees. ACL injuries occurred on average $23(17-28)$ years prior to testing (see Table 1 and our previous article [18] for more details regarding demographics, background data, surgery techniques, physiotherapy treatment, and the recruitment process). ACL-injured participants were recruited from two different hospitals in Sweden. For $\mathrm{ACL}_{\mathrm{R}}$, physiotherapy treatment was provided for prehabilitation purposes for 3 months before reconstructive surgery was performed along with post-operative physiotherapy of at least 22 weeks. ACL $\mathrm{AT}_{\mathrm{PT}}$ were treated solely with physiotherapy until specific screening tests could be performed sufficiently without instability or symptoms after a median time of 22 weeks (range 12-60 weeks). Exclusion criteria for the present study were bilateral ACL injury, other severe injury or disease to the non-injured leg, prosthesis, or any other musculoskeletal, rheumatological or neurological pathology. Controls were recruited through advertisement and convenience sampling and were matched to ACL-injured participants with regard to age and sex. In addition to self-reporting of asymptomatic knees, clinical examinations of controls were performed to exclude injury of

Table 1 Participant characteristics (mean (SD), unless otherwise stated)

\begin{tabular}{|c|c|c|c|}
\hline & \multicolumn{3}{|l|}{ Groups } \\
\hline & $\mathrm{ACL}_{\mathrm{R}}$ & ACLPT & CTRL \\
\hline Participants (n) & 33 & 37 & 33 \\
\hline Males/females ( $n$ ) & $21 / 12$ & $23 / 14$ & $21 / 12$ \\
\hline Age at test (years) & $45.6(4.5)$ & $48.1(5.9)$ & $46.7(5.0)$ \\
\hline Years since injury & $23.9(2.8)$ & $23.1(1.3)$ & - \\
\hline $\begin{array}{l}\text { Years between injury } \\
\text { and surgery }\end{array}$ & $3.6(2.3)$ & - & - \\
\hline Height (cm) & $174.0(9.1)$ & $173.5(8.0)$ & $176.4(9.8)$ \\
\hline Weight (kg) & $83.0(15.6)$ & $87.1(14.9)$ & $77.4(14.9)^{a}$ \\
\hline $\mathrm{BMI}\left(\mathrm{kg} / \mathrm{m}^{2}\right)$ & $27.2(3.3)$ & $28.9(4.6)$ & $24.6(2.5)^{\mathrm{b}}$ \\
\hline $\begin{array}{l}\text { Injury side: dominant/ } \\
\text { non-dominant }\end{array}$ & $21 / 12$ & 20/17 & - \\
\hline \multicolumn{4}{|l|}{ Cause of injury: } \\
\hline Soccer $(n)$ & 24 & 25 & - \\
\hline Alpine skiing $(n)$ & 2 & 5 & - \\
\hline Other sports $(n)$ & 6 & 2 & - \\
\hline Non-sport related $(n)$ & 1 & 5 & - \\
\hline $\mathrm{OA} \mathrm{K \& L^{c }} 1(n)$ & 5 & 6 & - \\
\hline OA K\&L $2(n)$ & 12 & 13 & - \\
\hline OA K\&L $3(n)$ & 10 & 9 & - \\
\hline OA K\&L $4(n)$ & 4 & 3 & - \\
\hline
\end{tabular}

Table adapted from Tengman et al. (15)

Abbreviations: BMI Body mass index, OA Osteoarthritis, SD Standard deviation Significant differences:

${ }^{\mathrm{a} A C L}$ PT vs. CTRL, $p=0.025$

${ }^{\mathrm{b}} \mathrm{ACL}_{\mathrm{R}}$ vs. CTRL, $p=0.014 ; \mathrm{ACL}_{\mathrm{PT}}$ vs. CTRL, $p<0.001$

'Radiographic OA was graded according to Kellgren \& Lawrence [19]; K\&L 0-

$1=$ no-or-low, K\&L 2-4 = moderate-to-high degree of OA the ACL, other ligaments, or the meniscus so that controls were deemed eligible for participation as controls in the study. All participants received prior information about the study before providing their written informed consent in accordance with the declaration of Helsinki. The study was approved by the Regional Ethical Review Board of Umeå, Sweden (Dnr. 08-211 M).

\section{Procedures and data collection}

The OLR was performed as part of a test battery consisting of nine different tests at the U-Motion laboratory, Umeå University, Sweden. Participants began the OLR by sitting on a stool (height $0.48 \mathrm{~m}$ ) and were asked to perform as many repetitive sit-stand-sit movements as possible at a self-selected but controlled speed with only 1 foot on the floor and arms across the chest. Participants continued to either failure or were stopped if achieving 50 repetitions in line with the protocol by Hart et al., [10], although they were unaware of this maximum prior to the test. Failure was defined if the contralateral non-weight bearing leg/foot contacted the weight-bearing leg or the ground or if the foot of the standing leg moved position. ACL-injured participants started with their non-injured leg and controls with their dominant leg (dominance defined as the leg preferred to kick a ball) after performing one practice repetition. The test was repeated with the contralateral leg after a minimum two-minute rest. An eight-camera three-dimensional motion capture system (Oqus Qualisys, Gothenburg, Sweden, $240 \mathrm{~Hz}$ ) and one two-dimensional video camera recorded all movements. Qualisys Track Manager software (version 2.2, Qualisys, Gothenburg, Sweden) was used to capture and track 42 retro-reflective markers which were affixed by double-sided adhesive tape to the skin of the participants on specific anatomical landmarks of the trunk and lower body according to an adapted Helen Hayes marker set, described in more detail in our previous article [20].

\section{Data analysis \\ Performance screening and analysis}

The performance outcome variable of the OLR test was defined as the total number of consecutive successful repetitions achieved for each respective leg. In this respect, one repetition of the OLR was defined as beginning when participants were no longer in contact with the stool and ended once participants next sat on the stool after having achieved a standing position, defined and controlled visually by the test leader during testing as a fully extended knee, on the tested leg. All repetitions were checked for adherence to the desired protocol firstly by the lead author using video footage and when uncertainty arose together with a co-author (ET). Unsuccessful trials, along with subsequent attempts, 
were omitted from all analyses. However, when participants performed the first repetition incorrectly and continued with successful attempts, the first repetition was omitted but subsequent successful repetitions were included.

\section{Data processing and reduction}

Marker trajectory data were gap-filled using polynomial interpolation in Qualisys Track Manager software when deemed accurate up to a maximum of 10 frames per sequence. Marker data were then exported to Visual3D software (Visual3D Professional version 5.02.23, C-Motion Inc., Germantown, Maryland, USA) and low-pass filtered using a second order Butterworth filter with a cut-off frequency of $6 \mathrm{~Hz}$. A fivesegment rigid-body model consisting of two shanks, two thighs and one pelvis was then constructed, with joint centres based on a 6-degrees-of-freedom model. Knee joint angles were defined as the rotation of the shank relative to the thigh using the Cardan X-Y-Z convention, so that (with positive rotations from zero presented first, i.e. positive/negative) $\mathrm{X}$ represented flexion/extension, Y represented adduction/abduction, and $\mathrm{Z}$ represented internal/external rotation [21]. We decomposed the OLR into four phases (Fig. 1) with start and end times determined by the vertical velocity of the hip joint centre, where positive values equate to an upwards direction. Maximum and minimum velocity were identified for each included repetition of each participant. Phases were thus defined as follows: i) Rise began when the hip joint centre velocity first exceeded $10 \%$ of its maximum, ii) Stand began when the hip joint centre velocity was next below $10 \%$ of its maximum, iii) Down began when the hip joint centre velocity was next below $10 \%$ of its minimum, and iv) Sit began when the hip joint centre velocity next exceeded $10 \%$ of its minimum. All events were checked manually and adjusted if considered incorrect.

\section{Kinematic analyses}

To capture representative knee kinematics during the OLR, the first successful repetition was omitted from kinematic analyses in line with a previous study [8] in order to avoid adjustments of body position. Thus, all consecutive successful repetitions from attempts 2 to 11 for each participant were analysed, resulting in up to 10 repetitions per participant. Ten repetitions were chosen to gain a reasonably representative analysis of movement patterns. Including more than 10 repetitions however, may have increased fatigue effects which have been shown to effect knee kinematics among ACL-reconstructed women [22]. We calculated the percentage time taken to complete each of the four movement phases within each repetition. For each Rise and Down phase we calculated maximum knee abduction and adduction angles, knee abduction/adduction range of motion, and mediolateral knee control. Mediolateral knee control was investigated by calculating the total number of knee movement units, based on a measure commonly used to assess arm reaching movements [23]. We operationally defined knee movement units as each knee velocity peak in the frontal plane on the knee velocity profile $>10 \%$ of the maximum knee velocity in the same direction. Means and standard deviations (SD) for maximum knee abduction, adduction, abduction/adduction range, and total movement units were subsequently calculated for the Rise and Down phases of all included repetitions for each individual.

\section{Statistical analyses}

Statistics were performed using IBM SPSS Statistics for Windows, version 23.0 (IBM Corp., Armonk, N.Y., USA). Outliers in the kinematic data $(2.93 \%$ of the total data set) were reviewed and corresponding repetitions were cross-checked using video and motion capture recordings. No outliers were deemed to be due to technical or data entry errors and therefore none were removed from the statistical analyses. Skewness and Shapiro-Wilk tests were performed to

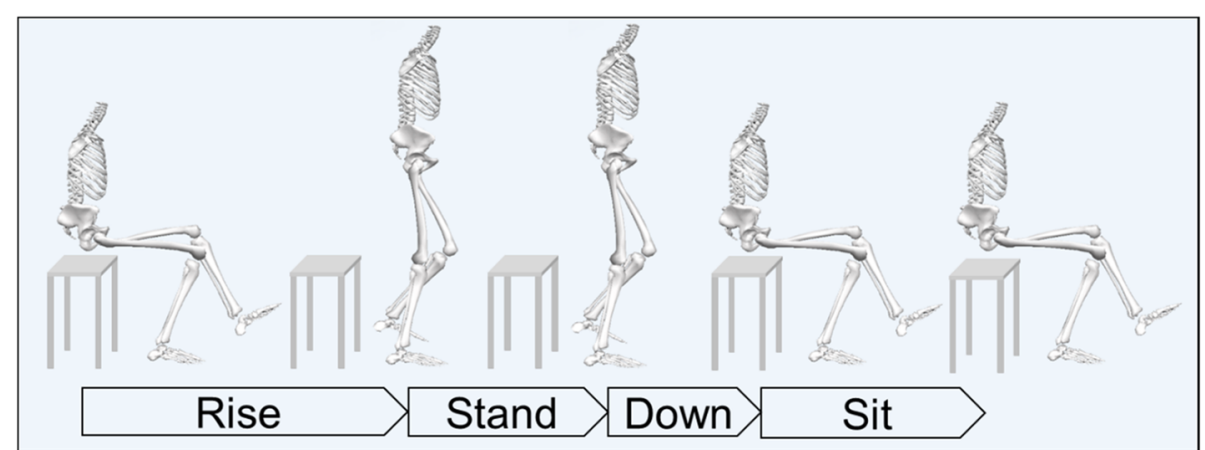

Fig. 1 A schematic illustration of the four defined phases for one repetition of the One-leg rise test. (Fig. 1 was conceived, designed and produced by the authors of this manuscript) 
assess data distributions. Subsequently, between-group comparisons of demographics for age, height, weight, and body mass index (BMI) were performed using One-way analysis of variance (ANOVA) tests due to normal data distributions and significant results followed up with Bonferroni post hoc tests. Betweengroup analyses of performance (number of repetitions and phase completion time) and knee kinematics compared ACL-injured legs to each other and to the non-dominant legs of CTRL as well as the noninjured legs of the ACL-injured groups to each other and to the dominant legs of CTRL, for a stringent comparison. Bland-Altman plots for kinematic variables were used to screen for systematic bias between repetition 2 and 11 [24]. All between-group performance and kinematic variables, including the LSI which was calculated for each individual by dividing the outcome measure for the injured or non-dominant leg by that of the non-injured or dominant leg respectively and multiplying by 100 , were analysed using non-parametric Kruskal Wallis tests due to nonnormally distributed data and significant results were followed up with Dunn-Bonferroni post hoc pairwise tests. Estimates of the effect sizes $(r)$ for significant between group comparisons were calculated using the Z statistic of Mann-Whitney U tests:

$$
r=\frac{\mathrm{Z}}{\sqrt{ } n}
$$

where $\mathrm{Z}=$ Mann-Whitney U Z statistic.

$n=$ the number of participants.

Cumulative percentages of completed repetitions were calculated and these distributions were statistically compared between groups using two-sample KolmogorovSmirnov tests. The total number of participants who completed 20 repetitions was compared between groups with Pearson's chi-squared test. Within-group comparisons compared between legs within each group using Wilcoxon Signed Ranks tests and the associated Z statistic. Estimates of the effect sizes $(r)$ for significant within-group comparisons were calculated using the $\mathrm{Z}$ statistic [25]:

$$
r=\frac{\mathrm{Z}}{\sqrt{n}}
$$

where $\mathrm{Z}=$ Wilcoxon Signed Ranks Z statistic.

$n=$ the number of pairs.

Effect sizes were considered large if 0.5 , medium if 0.3 and small if 0.1 [25]. Significance levels were set a priori $(\alpha=0.05)$. Within-session reliability was calculated for the knee kinematics of all groups and legs based on repetitions 2-11. Reliability was calculated for the following variables of the knee separately for both the Rise and Down phases: 1) maximum abduction, 2) maximum adduction, 3) maximum abduction/adduction range, and 4) movement units in the frontal plane. Intraclass Correlation Coefficient for a two-way mixed model where the mean of repeated measures (ICC 3,k) and absolute agreement was calculated [26].

ICC classification of reliability was made according to Fleiss [27], thus ICC $<0.40=$ poor, $\mathrm{ICC}>0.40$ but $<0.75=$ fair to good, and ICC $>0.75=$ excellent. The standard error of measurement (SEM) was calculated to provide an estimate of the error in the units of measurement, thus giving clinically relevant values for expected error in each individual. It was calculated as the square root of the mean square error term from the ANOVA [28].

\section{Results \\ Performance of the OLR test Total repetitions}

All participants were included in performance analyses for total repetitions. The number of participants completing the maximum 50 repetitions were for the ACL-injured/ CTRL non-dominant leg: $n=\mathrm{ACL}_{\mathrm{R}}$ 8, ACL $\mathrm{AT}_{\mathrm{PT}}$ 5, CTRL 12, and for the ACL non-injured/CTRL dominant leg: $n=$ $\mathrm{ACL}_{\mathrm{R}}$ 8, ACL $\mathrm{PT}_{\mathrm{PT}}$ 8, CTRL 16. Median (Quartile 1, Quartile 3) successful repetitions for the ACL-injured/CTRL nondominant leg were: $\mathrm{ACL}_{\mathrm{R}} 30(10,49), \mathrm{ACL}_{\mathrm{PT}} 15(6.5,33.5)$, CTRL 32 (12, 50), and for the ACL non-injured/CTRL dominant leg: $\mathrm{ACL}_{\mathrm{R}} 20(11,49.5), \mathrm{ACL}_{\mathrm{PT}} 21(3,39.5)$, and CTRL 37 (18, 50). ACL PT $_{\text {PT }}$ performed significantly fewer repetitions than CTRL for the injured leg compared to the non-dominant leg respectively $(r=-0.27, p=0.050)$. Distributions of cumulative percentages are displayed in Fig. 2 and were significantly different $(p<0.02)$ for all between group comparisons except for between the non-injured leg of $\mathrm{ACL}_{\mathrm{R}}$ and $A C L_{\mathrm{PT}}$. For the ACL-injured/CTRL nondominant leg comparisons, $59 \%$ of $\mathrm{ACL}_{\mathrm{PT}}$ were unable to complete 20 repetitions, compared to $33 \% \mathrm{ACL}_{\mathrm{R}}$ and $36 \%$ CTRL, although these between-group differences were not significant. No other between- or within-group differences were statistically significant for total repetitions. The number of participants unable to perform any repetitions for the ACL-injured/ CTRL non-dominant leg were: $n=\mathrm{ACL}_{\mathrm{R}}$ 2, $\mathrm{ACL}_{\mathrm{PT}} 3$, CTRL 2, and for the ACL non-injured/CTRL dominant leg: $n=\mathrm{ACL}_{\mathrm{R}} 2$, ACL $\mathrm{PT}_{\mathrm{PT}}$ 5, CTRL 0 . The LSI was calculated for each individual who had performed at least one repetition on each leg $\left(n=\mathrm{ACL}_{\mathrm{R}} 31, \mathrm{ACL}_{\mathrm{PT}} 32\right.$, CTRL 31) but was not significantly different between groups (medians (Quartile 1, Quartile 3)): $\mathrm{ACL}_{\mathrm{R}} 100$ (90.5, 157.1), $\mathrm{ACL}_{\mathrm{PT}} 100(57.9,104.8)$, CTRL $\left.100(67.7,100)\right)$.

\section{Time of completion - phase percent time}

Time of completion analyses, as well as for knee kinematics, required participants to complete more than one repetition of the OLR. Therefore, participants included in between-group comparisons were: ACL- 

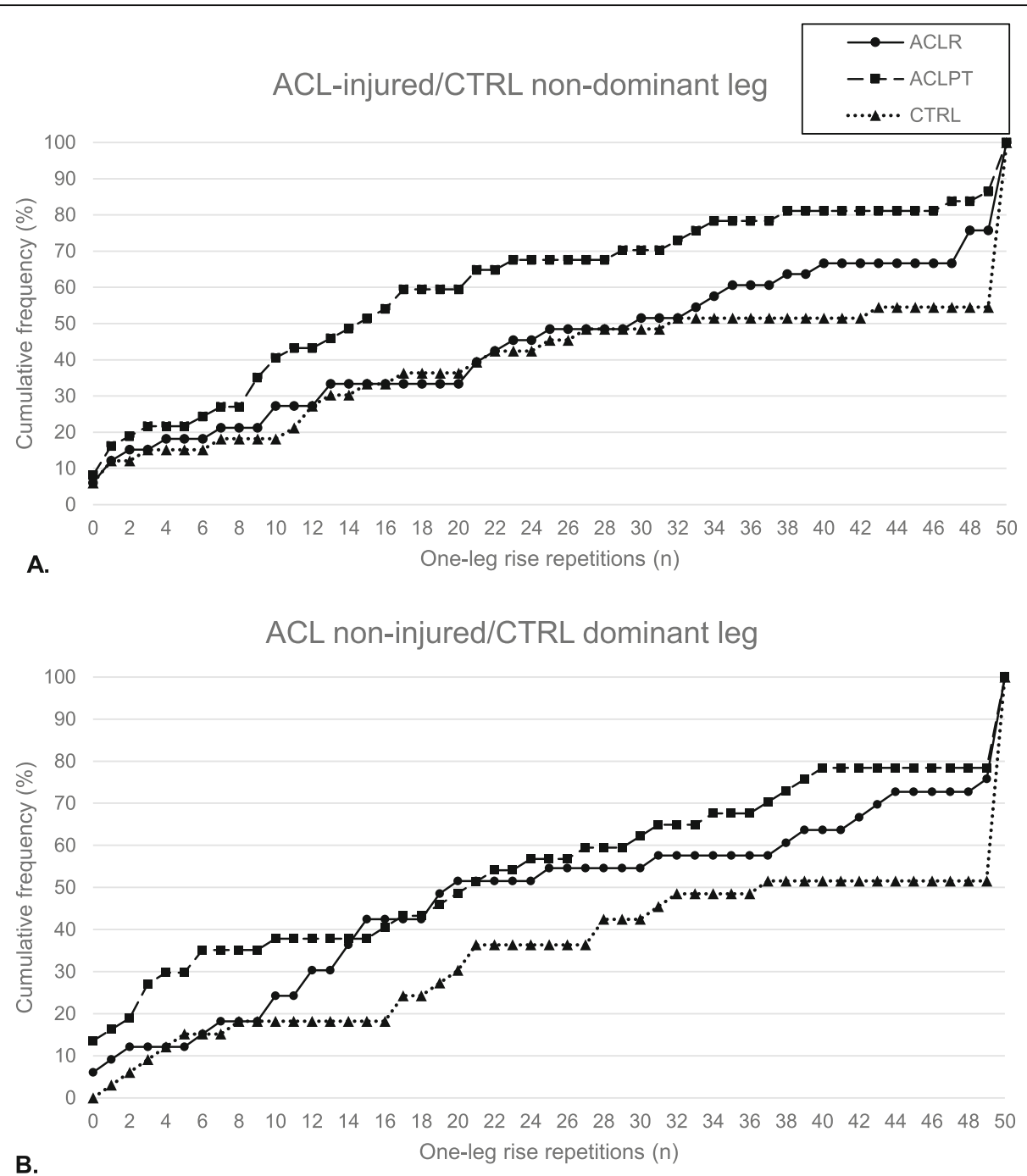

Fig. 2 Graphs displaying the amount of repetitions completed by all participants from each group as cumulative percentages clearly demonstrating the difference in distribution between groups. A) the non-injured leg of $A C L_{R}$ and $A C L_{p T}$ and the dominant leg of $C T R L$, and B) the injured leg of $A C L_{R}$ and $A C L_{p T}$ and the non-dominant leg of CTRL

injured/CTRL non-dominant leg, $n=\mathrm{ACL}_{\mathrm{R}} 29, \mathrm{ACL}_{\mathrm{PT}}$ 31, CTRL 29; ACL non-injured/CTRL dominant leg, $n=\mathrm{ACL}_{\mathrm{R}} 30, \mathrm{ACL}_{\mathrm{PT}}$ 31, CTRL 32. No between-group differences were statistically significant for percent time of phase completion (Table 2). Within-group (betweenleg) analyses for time completion, and for knee kinematics, required each participant to complete more than one repetition on both legs. As such, the number of participants included in this analysis was further reduced for $A C L_{P T}$ and thus included participants for within-group comparisons were: $n=\mathrm{ACL}_{\mathrm{R}} 29, \mathrm{ACL}_{\mathrm{PT}}$ 28, CTRL 29. ACL $L_{\mathrm{PT}}$ spent significantly longer in the Sit phase when using their non-injured leg compared to their injured leg (median difference $0.12 \mathrm{~s}, \mathrm{Z}=2.18, r=$ $0.41, p=0.03)$. CTRL took significantly longer to complete the Rise phase with their non-dominant leg compared to their dominant leg (median difference $0.62 \%, \mathrm{Z}=-2.53, r=-0.45, p=0.01$ ), but significantly longer to complete the Down phase with their dominant leg compared to their non-dominant leg (median difference $0.03 \mathrm{~s}, \mathrm{Z}=2.07, r=0.38, p=0.04$ ). All significant differences were thus of medium effect size and no other within-group differences were statistically significant for percent time of phase completion.

\section{Knee kinematic variables}

Included participants for kinematic analyses are stated above in the section Time of completion - phase percent time. Fig. 3 shows mean knee angle curves in the frontal plane for all groups throughout the Rise and Down phases for both legs in up to 10 repetitions per participant. During the Rise phase, $\mathrm{ACL}_{\mathrm{PT}}$ displayed on average $2.6^{\circ}$ greater knee abduction 
Table 2 Comparisons of normalised time (\%) and total time (sec) of completion for each phase of the One-leg-rise test for all groups and both legs. Values are group medians (Quartile 1, Quartile 3) related to between-group comparisons. Between-leg comparisons within groups were based on differences between legs of each individual

\begin{tabular}{|c|c|c|c|c|c|c|}
\hline \multirow{2}{*}{$\begin{array}{l}\text { Phases } \\
\text { and legs }\end{array}$} & \multicolumn{3}{|l|}{ Normalised time (\%) } & \multicolumn{3}{|l|}{ Total time (sec) } \\
\hline & $\mathrm{ACL}_{\mathrm{R}}$ & $A C L_{P T}$ & CTRL & $\mathrm{ACL}_{\mathrm{R}}$ & $A C L_{P T}$ & CTRL \\
\hline \multicolumn{7}{|l|}{ Rise } \\
\hline Inj/ND & $27.94(25.50,33.00)$ & $27.66(25.71,31.96)$ & $29.94(26.83,33.86)^{a}$ & $0.99(0.87,1.21)$ & $0.96(0.87,1.06)$ & $0.96(0.81,1.10)$ \\
\hline NI/Dom & $28.39(23.94,31.79)$ & $28.15(26.16,31.30)$ & $28.27(25.05,30.39)^{a}$ & $1.04(0.89,1.15)$ & $0.95(0.86,1.12)$ & $0.93(0.85,1.14)$ \\
\hline \multicolumn{7}{|l|}{ Stand } \\
\hline Inj/ND & $13.13(10.10,19.80)$ & $14.57(10.03,23.96)$ & $13.41(8.69,18.37)$ & $0.42(0.30,0.84)$ & $0.50(0.28,0.86)$ & $0.40(0.24,0.59)$ \\
\hline NI/Dom & $14.77(11.15,20.11)$ & $13.99(9.89,18.97)$ & $13.33(8.73,15.62)$ & $0.53(0.36,0.91)$ & $0.48(0.28,0.69)$ & $0.44(0.29,0.58)$ \\
\hline \multicolumn{7}{|l|}{ Down } \\
\hline $\operatorname{lnj} / \mathrm{ND}$ & $31.13(24.96,36.02)$ & $32.93(27.97,37.37)$ & $30.68(28.31,36.23)$ & $1.03(0.95,1.31)$ & $1.08(0.95,1.28)$ & $0.99(0.88,1.19)$ \\
\hline NI/Dom & $31.67(24.96,34.52)$ & $31.11(29.14,34.17)$ & $30.56(26.73,33.02)$ & $1.07(0.98,1.27)$ & $1.08(0.91,1.32)$ & $1.02(0.93,1.17)$ \\
\hline \multicolumn{7}{|l|}{ Sit } \\
\hline $\operatorname{lnj} / \mathrm{ND}$ & $29.48(23.28,33.57)$ & $24.39(17.75,29.11)$ & $27.43(24.88,32.04)$ & $0.97(0.75,1.31)$ & $0.78(0.65,1.02)^{b}$ & $0.91(0.68,1.17)$ \\
\hline NI/Dom & $27.08(18.58,32.52)$ & $27.33(20.01,30.39)$ & $28.80(25.42,33.20)$ & $0.88(0.64,1.41)$ & $0.90(0.66,1.10)^{b}$ & $1.02(0.87,1.21)$ \\
\hline
\end{tabular}

Bold text highlights significant differences

Abbreviations: Inj ACL-injured, ND CTRL non-dominant, NI ACL non-injured, Dom CTRL dominant

Significant within-group (between-leg) differences:

${ }^{a}$ CTRL Rise phase normalized time; dominant leg vs. non-dominant leg $(Z=-2.53, r=-0.45, p=0.01)$

${ }^{\mathrm{b}} A C L_{P T}$ Sit phase total time; injured leg vs. non-injured leg $(Z=2.18, r=0.41, p=0.03)$

${ }^{c} \mathrm{CTRL}$ Down phase total time; dominant leg vs. non-dominant leg $(Z=2.07, r=0.38, p=0.04)$.

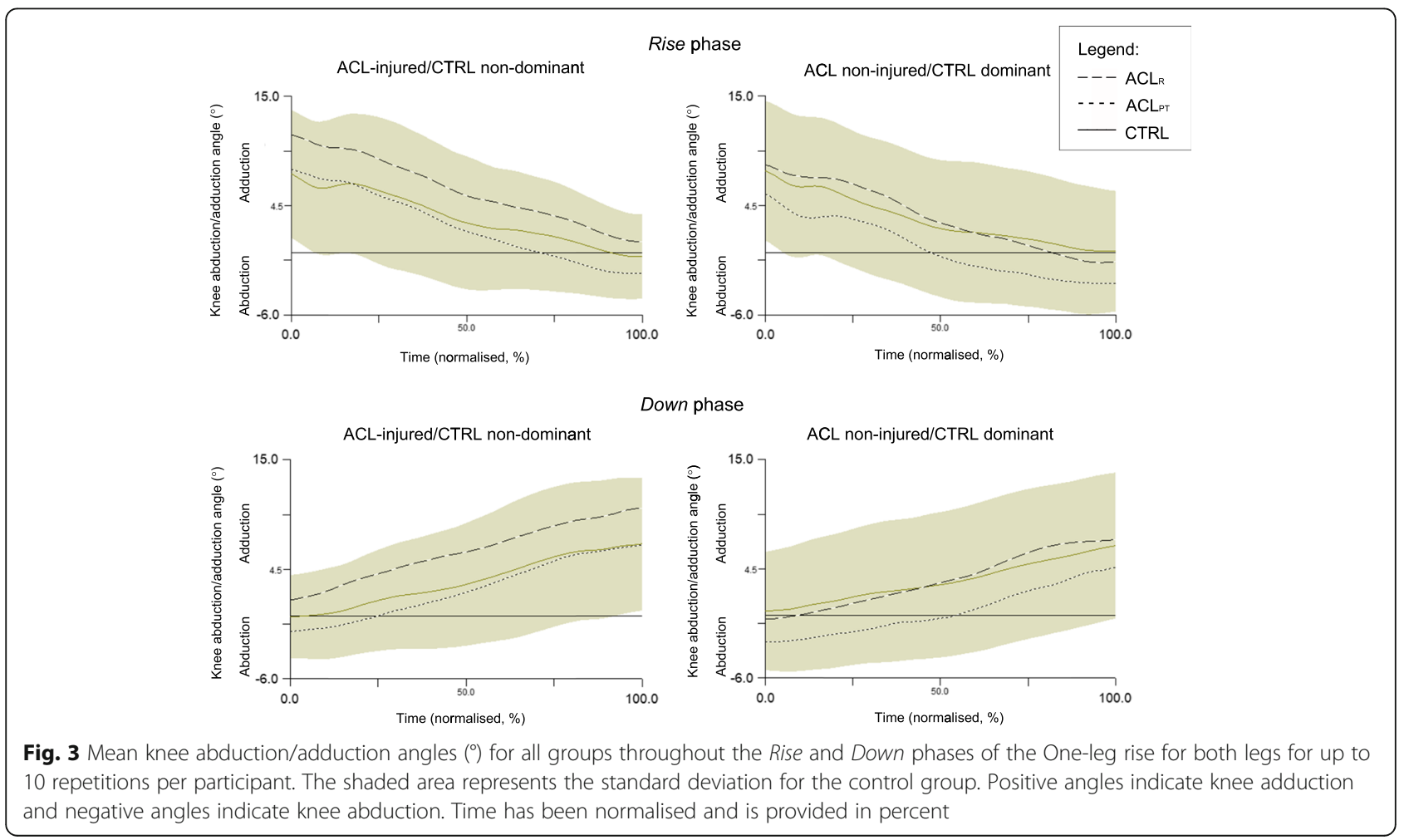


maximum than $\mathrm{ACL}_{\mathrm{R}}$ for their injured leg $(r=-0.33, p=$ $0.038)$ and $3.4^{\circ}$ greater for their non-injured leg $(r=-0.36$, $p=0.034$ ) as well as $3.6^{\circ}$ greater for their non-injured leg compared to the dominant leg of CTRL $(r=-0.32, p=$ 0.021) (Table 3). During the Down phase, $\mathrm{ACL}_{\mathrm{PT}}$ displayed on average $3.7^{\circ}$ greater knee abduction maximum for their injured leg compared to the injured leg of $\mathrm{ACL}_{\mathrm{R}}(r=-0.32$, $p=0.029)$ and $3.1^{\circ}$ greater for the non-injured leg compared to the dominant leg of CTRL $(r=-0.31, p=0.036)$. No between-group differences were statistically significant for knee adduction maximum, knee adduction/abduction range or knee movement units (Table 3). $\mathrm{ACL}_{\mathrm{R}}$ displayed on

Table 3 Between-group comparisons based on group medians (Quartile 1, Quartile 3) for knee abduction maximum, knee adduction maximum, knee abduction/adduction range, and knee movement units in the frontal plane during the Rise and Down phases of the One-leg rise. P-values $(p)$ and effect sizes $(r)$ are provided for all significant results

\begin{tabular}{|c|c|c|c|c|c|c|}
\hline \multirow[t]{2}{*}{ Variables } & \multicolumn{3}{|l|}{ Groups } & \multicolumn{3}{|c|}{ Group comparisons } \\
\hline & $\mathrm{ACL}_{\mathrm{R}}$ & $A C L_{P T}$ & CTRL & $\mathrm{ACL}_{\mathrm{R}}-\mathrm{ACL_{PT }}$ & $\mathrm{ACL}_{R}-\mathrm{CTRL}$ & $\mathrm{ACL}_{P T}-\mathrm{CTRL}$ \\
\hline \multicolumn{7}{|l|}{ Rise phase } \\
\hline \multicolumn{7}{|l|}{$\begin{array}{l}\text { Knee abd } \\
\max \left({ }^{\circ}\right)\end{array}$} \\
\hline Inj/ND & $0.51(2.47,-2.67)$ & $3.07(7.47,-0.35)$ & $0.68(5.00,-1.55)$ & $\begin{array}{l}p=0.038 \\
r=-0.33\end{array}$ & NS & NS \\
\hline NI/Dom & $1.67(4.85,-1.08)$ & $5.03(6.52,2.69)$ & $1.40(6.25,-3.13)$ & $\begin{array}{l}p=0.034 \\
r=-0.36\end{array}$ & NS & $\begin{array}{l}p=0.021 \\
r=-0.32\end{array}$ \\
\hline \multicolumn{7}{|l|}{$\begin{array}{l}\text { Knee add } \\
\max \left({ }^{\circ}\right)\end{array}$} \\
\hline $\operatorname{lnj} / N D$ & $10.14(7.49,15,67)$ & $7.94(3.85,15.45)$ & $8.27(4.29,12.89)$ & NS & NS & NS \\
\hline NI/Dom & $8.99(6.74,12.17)$ & $6.51(0.95,9.82)$ & $6.82(2.96,14.35)$ & NS & NS & NS \\
\hline \multicolumn{7}{|c|}{$\begin{array}{l}\text { Knee abd/add } \\
\text { range }\left(\left(^{\circ}\right)\right.\end{array}$} \\
\hline $\operatorname{lnj} / N D$ & $11.40(7.74,14.57)$ & $12.30(7.48,15.87)$ & $10.54(7.27,12.93)$ & NS & NS & NS \\
\hline NI/Dom & $11.00(8.54,13.33)$ & $10.49(8.08,15.16)$ & $8.85(6.94,12.10)$ & NS & NS & NS \\
\hline \multicolumn{7}{|c|}{ Knee MU (n) } \\
\hline $\operatorname{lnj} / \mathrm{ND}$ & $4.67(4.35,5.75)$ & $5.00(4.17,5.33)$ & $5.00(4.30,5.70)$ & NS & NS & NS \\
\hline NI/Dom & $5.20(4.68,6.00)$ & $5.10(4.22,5.45)$ & $4.60(4.20,5.30)$ & NS & NS & NS \\
\hline \multicolumn{7}{|l|}{ Down phase } \\
\hline \multicolumn{7}{|l|}{$\begin{array}{l}\text { Knee abd } \\
\max \left({ }^{\circ}\right)\end{array}$} \\
\hline Inj/ND & $-0.56(2.20,-4.24)^{a}$ & $3.13(6.65,-1.39)$ & $0.67(5.06,-1.70)$ & $\begin{array}{l}p=0.029 \\
r=-0.32\end{array}$ & NS & NS \\
\hline NI/Dom & $0.48(4.64,-2.23)^{a}$ & $4.04(6.43,2.41)$ & $0.96(5.30,-3.68)$ & NS & NS & $\begin{array}{l}p=0.036 \\
r=-0.31\end{array}$ \\
\hline \multicolumn{7}{|l|}{$\begin{array}{l}\text { Knee add } \\
\max \left({ }^{\circ}\right)\end{array}$} \\
\hline $\operatorname{lnj} / \mathrm{ND}$ & $9.24(6.52,15.42)^{b}$ & $8.03(2.95,13.04)$ & $9.25(3.84,11.83)$ & NS & NS & NS \\
\hline NI/Dom & $7.29(5.48,11.95)^{b}$ & $4.80(-0.06,8.95)$ & $5.78(1.91,13.81)$ & NS & NS & NS \\
\hline \multicolumn{7}{|c|}{$\begin{array}{l}\text { Knee abd/add } \\
\text { range }\left({ }^{\circ}\right)\end{array}$} \\
\hline Inj/ND & $8.90(6.41,13.50)$ & $9.57(6.73,14.30)$ & $8.57(6.09,10.76)$ & NS & NS & NS \\
\hline NI/Dom & $8.08(5.97,12.18)$ & $9.60(5.80,12.25)$ & $7.16(5.58,10.57)$ & NS & NS & NS \\
\hline \multicolumn{7}{|c|}{ Knee MU (n) } \\
\hline Inj/ND & $4.90(3.95,5.70)$ & $5.50(4.70,6.25)$ & $4.60(3.90,5.60)$ & NS & NS & NS \\
\hline NI/Dom & $4.80(4.28,5.65)$ & $5.15(4.28,6.10)$ & $4.70(4.08,5.35)$ & NS & NS & NS \\
\hline
\end{tabular}

Bold text highlights significant differences

Abbreviations: abd Abduction, add Adduction; Inj ACL-injured, ND CTRL non-dominant, NI ACL non-injured, Dom CTRL dominant, NS Not statistically significant, MU Movement units

Significant within-group (between-leg) differences,

${ }^{a}$ Knee abduction maximum for $\mathrm{ACL}_{\mathrm{R}}$ during Down phase; injured leg vs. non-injured leg, $p=0.035, r=-0.39$

${ }^{b}$ Knee adduction maximum for $\mathrm{ACL}_{\mathrm{R}}$ during Down phase; injured leg vs. non-injured leg, $p=0.041, r=-0.38$ 
average $1.04^{\circ}$ greater knee abduction maximum in their noninjured leg compared to their injured leg during the Down phase $(\mathrm{Z}=-2.11, r=-0.39, p=0.035)$. Further, $\mathrm{ACL}_{\mathrm{R}}$ displayed on average $1.95^{\circ}$ greater knee adduction maximum in their injured leg than their non-injured leg during the Down phase $(\mathrm{Z}=-2.04, r=-0.38, p=0.041)$. No within-group differences were evident for knee abduction/adduction range or knee movement units (see Table 3).

\section{Within-session reliability of knee kinematics}

All knee kinematic variables showed excellent withinsession reliability for all groups and legs $\operatorname{ICC}(3,10)$
0.81-1.00, 95\% CI 0.67-1.00, SEM 0.93-1.95) during both the Rise and Down phase (Table 4).

\section{Discussion}

ACL-injured persons treated solely with physiotherapy performed significantly fewer OLR repetitions than age- and sex-matched persons with asymptomatic knees when using their injured and non-dominant leg respectively, albeit with a small effect size. The distribution of cumulative repetitions for ACL-injured/CTRL non-dominant leg comparisons revealed that $59 \%$ of $\mathrm{ACL}_{\mathrm{PT}}$ were unable to achieve the 20 repetition cut-off for predicting knee OA development

Table 4 Within-session reliability of the knee kinematic variables during One-leg rise test performance for all groups

\begin{tabular}{|c|c|c|c|c|c|c|c|c|c|}
\hline \multirow[t]{2}{*}{ Variables } & \multicolumn{9}{|l|}{ Groups } \\
\hline & \multicolumn{3}{|l|}{$\overline{A C L_{R}}$} & \multicolumn{3}{|l|}{$A C L_{P T}$} & \multicolumn{3}{|l|}{ CTRL } \\
\hline \multicolumn{10}{|l|}{ Rise phase } \\
\hline & $\operatorname{ICC}(3, \mathrm{k})$ & $95 \% \mathrm{Cl}$ & SEM & $\operatorname{ICC}(3, \mathrm{k})$ & $95 \% \mathrm{Cl}$ & SEM & ICC $(3, k)$ & $95 \% \mathrm{Cl}$ & SEM \\
\hline \multicolumn{10}{|c|}{ Knee abd max $\left({ }^{\circ}\right)$} \\
\hline Inj/ND & 0.99 & $0.99-1.00$ & 1.03 & 0.99 & $0.99-1.00$ & 1.21 & 1.00 & $0.99-1.00$ & 1.04 \\
\hline NI/Dom & 0.99 & $0.99-1.00$ & 1.04 & 0.99 & $0.99-1.00$ & 1.17 & 0.99 & $0.99-1.00$ & 1.37 \\
\hline \multicolumn{10}{|c|}{ Knee add max $\left(^{\circ}\right)$} \\
\hline Inj/ND & 1.00 & $0.99-1.00$ & 1.45 & 1.00 & $0.99-1.00$ & 1.57 & 0.99 & $0.99-1.00$ & 1.30 \\
\hline NI/Dom & 0.99 & $0.98-1.00$ & 1.45 & 1.00 & $0.99-1.00$ & 1.31 & 1.00 & $0.99-1.00$ & 1.04 \\
\hline \multicolumn{10}{|c|}{$\begin{array}{l}\text { Knee abd/add } \\
\text { range }\left({ }^{\circ}\right)\end{array}$} \\
\hline $\operatorname{lnj} / \mathrm{ND}$ & 0.99 & $0.98-1.00$ & 1.86 & 0.99 & $0.98-1.00$ & 1.85 & 0.98 & $0.96-0.99$ & 1.57 \\
\hline NI/Dom & 0.99 & $0.98-0.99$ & 1.69 & 0.99 & $0.98-1.00$ & 1.64 & 0.97 & $0.95-0.99$ & 1.59 \\
\hline \multicolumn{10}{|l|}{ Knee MU (n) } \\
\hline Inj/ND & 0.86 & $0.76-0.93$ & 1.05 & 0.93 & $0.88-0.97$ & 1.24 & 0.87 & $0.78-0.93$ & 1.06 \\
\hline NI/Dom & 0.81 & $0.67-0.91$ & 1.13 & 0.86 & $0.74-0.93$ & 0.98 & 0.86 & $0.76-0.93$ & 1.05 \\
\hline \multicolumn{10}{|l|}{ Down phase } \\
\hline & ICC $(3, k)$ & $95 \% \mathrm{Cl}$ & SEM & $\operatorname{ICC}(3, k)$ & $95 \% \mathrm{Cl}$ & SEM & ICC $(3, k)$ & $95 \% \mathrm{Cl}$ & SEM \\
\hline \multicolumn{10}{|c|}{ Knee abd max $\left(^{\circ}\right)$} \\
\hline Inj/ND & 1.00 & $0.99-1.00$ & 0.93 & 0.99 & $0.99-1.00$ & 1.46 & 0.99 & $0.99-1.00$ & 1.06 \\
\hline NI/Dom & 0.99 & $0.99-1.00$ & 0.93 & 1.00 & $0.99-1.00$ & 0.93 & 1.00 & $0.99-1.00$ & 1.29 \\
\hline \multicolumn{10}{|c|}{ Knee add $\max \left({ }^{\circ}\right)$} \\
\hline $\operatorname{lnj} / N D$ & 0.99 & $0.99-1.00$ & 1.60 & 1.00 & $0.99-1.00$ & 1.57 & 0.99 & $0.99-1.00$ & 1.44 \\
\hline NI/Dom & 0.99 & $0.98-1.00$ & 1.51 & 1.00 & $0.99-1.00$ & 1.34 & 1.00 & $0.99-1.00$ & 0.99 \\
\hline \multicolumn{10}{|c|}{$\begin{array}{l}\text { Knee abd/add } \\
\text { range }\left({ }^{\circ}\right)\end{array}$} \\
\hline Inj/ND & 0.99 & $0.98-1.00$ & 1.83 & 0.98 & $0.97-0.99$ & 1.95 & 0.98 & $0.97-0.99$ & 1.59 \\
\hline NI/Dom & 0.99 & $0.98-1.00$ & 1.71 & 0.99 & $0.98-0.99$ & 1.62 & 0.98 & $0.97-0.99$ & 1.52 \\
\hline \multicolumn{10}{|l|}{ Knee MU (n) } \\
\hline Inj/ND & 0.87 & $0.78-0.94$ & 1.15 & 0.90 & $0.82-0.95$ & 1.43 & 0.92 & $0.87-0.96$ & 1.10 \\
\hline NI/Dom & 0.87 & $0.78-0.94$ & 1.29 & 0.85 & $0.74-0.93$ & 1.21 & 0.89 & $0.81-0.94$ & 1.38 \\
\hline
\end{tabular}


stated by Thorstensson et al., (2004) compared to $33 \% \mathrm{ACL}_{\mathrm{R}}$ and 36\% CTRL, although these differences were not statistically significant. $\mathrm{ACL}_{\mathrm{PT}}$ also displayed significantly greater knee abduction of medium effect sizes than both $\mathrm{ACL}_{\mathrm{R}}$ and CTRL during the Rise and Down phases of the OLR. Despite this, our findings showed inconsistent differences, particularly of knee kinematics, when comparing the ACL-injured groups to CTRL. This contradicted our previous research which found negative outcomes for the same ACL groups when compared to CTRL with regard to reduced control of single-limb stance [29], lower self-reported knee function and hop/jump capacity [18], and reduced knee muscle strength [30], as well as altered movement patterns during hop tests $[20,31,32]$. Thus the knee kinematics during the OLR, as performed and analysed in our study, did not discriminate certain existing disparities in knee movement control in the very long term after ACL injury.

Nevertheless, there was greater maximal knee abduction among $\mathrm{ACL}_{\mathrm{PT}}$ compared to $\mathrm{ACL}_{\mathrm{R}}$ and CTRL, although the differences were rather small but still significantly different. These differences in knee abduction align with a previous study of the same groups during landings from one-leg hops [20], although the clinical relevance in relation to detectable change remains to be determined. This finding is however further supported by Zhang and colleagues [33] who found greater knee abduction among ACL-deficient persons on average 5 years after injury compared to controls at heel contact during gait. Trulsson et al., [15] observed a greater medial position of the knee relative to the foot among nonoperated ACL-injured persons compared to controls when performing a battery of tests including a mini-squat. That said, a more medial position of the knee would not necessarily result in knee abduction, which is more specifically defined by rotation of the shank relative to the thigh. The greater knee abduction for the non-injured leg of $\mathrm{ACL}_{\mathrm{R}}$ in our study compared to their injured leg during the Down phase indicates even bilateral effects of the ACL injury. Indeed, reduced balance during a single-leg stance for both legs was previously seen for our $\mathrm{ACL}_{\mathrm{R}}$ and $\mathrm{ACL}_{\mathrm{PT}}$ groups [29]. Culvenor et al., [34] also reported reduced postural control 12 months post- $\mathrm{ACL}_{\mathrm{R}}$ for both legs when performing single-leg squats. One possible explanation for these bilateral effects may be neuroplastic changes following ACL injury, of which there is growing evidence $[35,36]$.

Advantages of the OLR include its convenience due to the lack of required equipment or space. The consistent stool height $(0.48 \mathrm{~m})$ used in our study is similar to that which is encountered daily and enhances ecological validity. The movement itself resembles the everyday task of standing and sitting which can provide a relevant evaluation of an individual's independence while isolating performance between legs. However, despite requiring more muscular effort than two-legged closed kinetic chain exercises, the OLR has been shown not to produce greater strains on the ACL than such tasks and can be considered appropriate for ACL-injured persons who can perform, for example, a traditional two-legged squat [37]. Further, the relative simplicity of the OLR compared to, e.g. a one-leg hop for distance, improves feasibility among populations of different ages and conditions. Nevertheless, the OLR requires adequate lower limb strength and endurance, coordination, balance and proprioceptive ability, factors which deteriorate across the life span. The OLR thus encompasses a number of important outcome variables for assessment of movement control. Furthermore, within-session reliability of our knee kinematic variables was excellent for all groups and legs, thus indicating that the observed movement patterns of these groups are consistent during repetitions 2-11 of the OLR and that averaged values are likely representative of each individual. This was also supported by the lack of systematic bias seen in Bland-Altman plots for these variables. Our proposal for assessing mediolateral knee control based on knee movement units revealed neither between- nor within-group differences for our comparisons in the present task. A similar movement control measure of the knee denominated fluency, defined as the number of times the velocity of the knee position in the coronal plane crossed zero when averaged per second, has however revealed worse mediolateral knee control among ACLinjured persons compared to controls during a one-leg hop for distance [16]. It is thus possible that our measure of knee movement units may discriminate movement control disparities in other more demanding tests and among populations with more severe pathologies and warrants further investigation.

Limitations of our study include the maximum 50 repetitions, applied to reduce fatigue effects on between-leg comparisons as well as the extreme delayed onset muscle soreness evident during pilot testing with no maximum. Statistically this created a ceiling effect and results would likely have been different without this maximum considering that 34 of 106 participants completed 50 repetitions on at least one leg and that CTRL accounted for 16 of those. Further, up to 229 repetitions were achieved in a previous study of chronic knee pain sufferers of similar age [7]. Additionally, the LSI was not an appropriate measure due to the maximum repetition limit and for those unable to perform a repetition on at least one leg. The determination of leg dominance, used to provide the most stringent comparison to controls by comparing the hypothesised less-competent and more competent legs separately between groups, i.e. ACLinjured vs. CTRL non-dominant and vice versa, was made according to which leg the participants preferred to kick a ball. However, recent evidence shows that 
certain healthy individuals change leg preference depending on the task involved [38], which may also be true for the OLR and for some injured persons. Thus, whether or not our between-group analysis resulted in the most stringent comparisons regarding injury side and dominance remains unclear. Our cross-sectional study design with long-term follow-up means that treatment strategies for ACL injuries have evolved since our participants were injured. Thus, our specific results may not be relevant for all ACL-injured persons. Other confounding factors across the two decades since injury such as, e.g. physical activity level, are also likely to have affected the outcome measures. We used $10 \%$ of the maximum/minimum hip joint centre velocity as a threshold level for setting the start/stop events of the OLR phases. Due to the lack of previous research investigating OLR kinematics, this decision was based on our own testing of various threshold levels across a number of participants and repetitions. Although we deemed this threshold level to be more appropriate than the alternatives that we tested, it is possible that choosing another threshold level may have changed the outcome of the results and thus further research is required to establish the most appropriate method. Further, there are common technical limitations to three-dimensional analyses, such as visibility of markers (hip and foot markers were often obscured when participants leaned forwards and due to the stool, respectively) or soft tissue artefacts which we tried to minimize using cluster markers and placement on solid anatomical landmarks [39]. The use of maximum values for kinematic variables is also sensitive to such artefacts and thus as well as data filtering, thorough manual checks were performed on movement profiles and data values in an attempt to ensure representative data.

Our study is the first to evaluate reliability of knee kinematics during execution of the OLR as well as implement the test to compare between legs of ACL-injured persons in the very long term after injury and to controls with asymptomatic knees. In future, adjustments to our protocol may help to improve the standardisation and discriminative ability of the OLR, which may lead to more successful application within research and clinics. Removing the maximum repetition limit, for example, appears feasible and should benefit interpretation. However, this may take a rather long time for completion, depending on the patient's functional state, which may make it less feasible for application in clinical settings. Further, standardisation regarding performance speed, e.g. using a metronome, may be considered. Randomisation of leg order in research studies would also help to avoid potential fatigue bias. The addition of kinetic data to enable analysis of body centre of pressure and joint moments is likely to provide valuable biomechanical information. Although advanced three-dimensional analysis was used in this study, if specific key movement control outcome variables can be identified, the use of simpler and less expensive video and software solutions may add value to clinical implementation of the OLR. Further reliability analysis should establish the minimum number of OLR repetitions required to provide reliable knee kinematic data, fatigue effects and additional pathological groups.

\section{Conclusions}

As long as two decades after injury, ACL-injured persons treated solely with physiotherapy performed fewer OLR repetitions than age- and sex-matched persons with asymptomatic knees when comparing the injured to the non-dominant leg respectively. The OLR also revealed greater knee abduction angles for $\mathrm{ACL}_{\mathrm{PT}}$ compared to $A C L_{R}$ and CTRL, indicating residual abnormal lower limb movement patterns. These results should however be interpreted with caution with regards to the potential treatment effects due to the very long time since injury and because this was not a randomised controlled study. The within-session reliability of the knee kinematics during the OLR among asymptomatic and ACL-injured knees was excellent and thus these measures are worth further exploration for use in research and clinics. Development of the OLR protocol and analysis methods may further improve its discriminative ability in identifying reduced knee function and abnormal movement patterns in research and clinical practice among a range of populations.

\section{Abbreviations}

Abd: Abduction; ACL: Anterior cruciate ligament; $A C L_{P T}$ : Anterior cruciate ligament physiotherapy-only treated group; $A C L_{R}$ : Anterior cruciate ligament reconstruction-treated group; Add: Adduction; ANOVA: Analysis of variance; BMI: Body mass index; Cl: Confidence intervals; CTRL: Control group; Dom: CTRL dominant; ICC: Intraclass correlation coefficient; Inj: ACL-injured; KACL2O-study: Knee injury - Anterior Cruciate Ligament after more than 20 years; LSI: Limb symmetry index; MU: Movement units; ND: CTRL nondominant; NI: ACL non-injured; OA: Osteoarthritis; OLR: One-leg rise; SEM: Standard error of measurement

\section{Acknowledgements}

Many thanks to Monica Edström for assistance in data collection, Jonas Selling for technical assistance, and to all of our participants.

\section{Authors' contributions}

$\mathrm{CKH}$ and ET conceived and designed the study. CKH procured the financing for the study. ET participated in the data acquisition. AS, ET, DS and CKH interpreted the data. AS performed the analysis and drafted the manuscript. $A S, E T, D S$ and $C K H$ performed critical in-depth reviews of the manuscript for intellectual content. All authors read and approved the final manuscript.

\section{Funding}

This study was funded by the Swedish Scientific Council (Grant No. K201499X-21876-04-4, Häger), King Gustaf V:s and Queen Victoria's Freemason's Foundation, Umeå University, Västerbotten County Council (Grant No. ALFVLL217301, VLL358901), Swedish Scientific Research Council for Sports Science, Umeå University School of Sport Science, and IngaBritt and Arne Lundberg's Research Foundation (Grant No. 363). Our funding sources had no involvement in the study design; in the collection, analysis, and interpretation of data; in the writing of the report; and in the decision to submit the article for publication. 


\section{Availability of data and materials}

The datasets used and/or analysed during the current study are available from the corresponding author on reasonable request.

\section{Ethics approval and consent to participate}

All participants received prior information about the study before providing their written informed consent in accordance with the declaration of Helsinki. The study was approved by the Regional Ethical Review Board of Umeå, Sweden (registration number 08-211 M).

\section{Consent for publication}

Not applicable.

\section{Competing interests}

All authors declare that they have no competing interests.

\section{Author details}

'Department of Community Medicine and Rehabilitation, Physiotherapy, Umeå University, SE-90187 Umeå, Sweden. ${ }^{2}$ Department of Industrial and Systems Engineering, Virginia Polytechnic Institute and State University, Blacksburg, VA, USA.

\section{Received: 25 April 2019 Accepted: 10 October 2019}

Published online: 25 October 2019

\section{References}

1. Suter LG, Smith SR, Katz JN, Englund M, Hunter DJ, Frobell R, Losina E. Projecting lifetime risk of symptomatic knee osteoarthritis and total knee replacement in individuals sustaining a complete anterior cruciate ligament tear in early adulthood. Arthritis Care Res (Hoboken). 2017:69:201-8.

2. Wiggins AJ, Grandhi RK, Schneider DK, Stanfield D, Webster KE, Myer GD. Risk of secondary injury in younger athletes after anterior cruciate ligament reconstruction: A systematic review and meta-analysis. Am J Sports Med. 2016:44:1861-76

3. Adams D, Logerstedt DS, Hunter-Giordano A, Axe MJ, Snyder-Mackler L. Current concepts for anterior cruciate ligament reconstruction: a criterion-based rehabilitation progression. J Orthop Sports Phys Ther. 2012:42:601-14.

4. Wellsandt E, Failla MJ, Snyder-Mackler L. Limb symmetry indexes can overestimate knee function after anterior cruciate ligament Injury. J Orthop Sports Phys Ther. 2017:47:334-8.

5. Zwolski C, Schmitt LC, Thomas S, Hewett TE, Paterno MV. The utility of limb symmetry indices in return-to-sport assessment in patients with bilateral anterior cruciate ligament reconstruction. Am J Sports Med. 2016:44:2030-8

6. van Melick N, van Cingel REH, Brooijmans F, Neeter C, van Tienen T, Hullegie W, der Sanden MWG N-v. Evidence-based clinical practice update: practice guidelines for anterior cruciate ligament rehabilitation based on a systematic review and multidisciplinary consensus. Br J Sports Med. 2016;50:1506.

7. Thorstensson CA, Petersson IF, Jacobsson LT, Boegard TL, Roos EM. Reduced functional performance in the lower extremity predicted radiographic knee osteoarthritis five years later. Ann Rheum Dis. 2004;63:402-7.

8. Thorstensson CA, Henriksson M, von Porat A, Sjodahl C, Roos EM. The effect of eight weeks of exercise on knee adduction moment in early knee osteoarthritis--a pilot study. Osteoarthr Cartil. 2007;15:1163-70.

9. Roos PE, Button K, van Deursen RW. Motor control strategies during double leg squat following anterior cruciate ligament rupture and reconstruction: an observational study. J Neuroeng Rehabil. 2014;11:19.

10. Hart HF, Collins NJ, Ackland DC, Crossley KM. Is impaired knee confidence related to worse kinesiophobia, symptoms, and physical function in people with knee osteoarthritis after anterior cruciate ligament reconstruction? I Sc Med Sport. 2015;18:512-7.

11. Culvenor AG, Lai CC, Gabbe BJ, Makdissi M, Collins NJ, Vicenzino B, Morris $\mathrm{HG}$, Crossley KM. Patellofemoral osteoarthritis is prevalent and associated with worse symptoms and function after hamstring tendon autograft ACL reconstruction. Br J Sports Med. 2014;48:435-9.

12. Riemann BL, Lephart SM. The sensorimotor system, part I: the physiologic basis of functional joint stability. J Athl Train. 2002;37:71-9.
13. Lohmander LS, Englund PM, Dahl LL, Roos EM. The long-term consequence of anterior cruciate ligament and meniscus injuries: osteoarthritis. Am J Sports Med. 2007;35:1756-69.

14. Yamazaki J, Muneta $T$, Ju YJ, Sekiya I. Differences in kinematics of single leg squatting between anterior cruciate ligament-injured patients and healthy controls. Knee Surg Sports Traumatol Arthrosc 2010;18:56-63.

15. Trulsson A, Garwicz M, Ageberg E. Postural orientation in subjects with anterior cruciate ligament injury: development and first evaluation of a new observational test battery. Knee Surg Sports Traumatol Arthrosc. 2010:18:814-23.

16. Roos PE, Button K, Sparkes V, van Deursen RW. Altered biomechanical strategies and medio-lateral control of the knee represent incomplete recovery of individuals with injury during single leg hop. J Biomech. 2014:47:675-80.

17. Cronstrom A, Ageberg E. Association between sensory function and medio-lateral knee position during functional tasks in patients with anterior cruciate ligament injury. BMC Musculoskelet Disord. 2014;15:430

18. Tengman E, Brax Olofsson L, Nilsson KG, Tegner Y, Lundgren L, Hager CK. Anterior cruciate ligament injury after more than 20 years: I. Physical activity level and knee function. Scand J Med Sci Sports. 2014:24:e491-500.

19. Kellgren JH, Lawrence JS. Radiological assessment of osteo-arthrosis. Ann Rheum Dis. 1957:16:494-502.

20. Tengman E, Grip H, Stensdotter A, Hager CK. Anterior cruciate ligament injury about 20 years post-treatment: A kinematic analysis of one-leg hop. Scand J Med Sci Sports. 2015;25:818-27.

21. Grip $\mathrm{H}$, Hager $\mathrm{C}$. A new approach to measure functional stability of the knee based on changes in knee axis orientation. J Biomech. 2013; 46:855-62.

22. Lessi GC, Silva RS, Serrao FV. Comparison of the effects of fatique on kinematics and muscle activation between men and women after anterior cruciate ligament reconstruction. Phys Ther Sport. 2018:31:29-34.

23. Von Hofsten C. Development of visually directed reaching: the approach phase. J Hum Mov Stud. 1979:5:160-78.

24. Bland JM, Altman DG. Statistics notes: Measurement error. BMJ. 1996;312:1654.

25. Fritz CO, Morris PE, Richler JJ. Effect size estimates: current use, calculations, and interpretation. J Exp Psychol Gen. 2012;141:2-18.

26. Trevethan R. Intraclass correlation coefficients: clearing the air, extending some cautions, and making some requests. Health Serv Outcome Res Methodol. 2017:17:127-43.

27. Fleiss J. The design and analysis of clinical experiments. New York: Wiley; 1986.

28. Weir JP. Quantifying test-retest reliability using the intraclass correlation coefficient and the SEM. J Strength Cond Res. 2005;19:231-40.

29. Stensdotter A-K, Tengman E, Olofsson LB, Häger C. Deficits in single-limb stance more than 20 years after ACL injury. Eur J Phys. 2013;15:78-85.

30. Tengman E, Brax Olofsson L, Stensdotter AK, Nilsson KG, Hager CK Anterior cruciate ligament injury after more than 20 years. II. Concentric and eccentric knee muscle strength. Scand J Med Sci Sports. 2014:24:e501-9.

31. Markstrom JL, Tengman E, Hager CK. ACL-reconstructed and ACL-deficien individuals show differentiated trunk, hip, and knee kinematics during vertical hops more than 20 years post-injury. Knee Surg Sports Traumatol Arthrosc. 2018:26:358-67.

32. Srinivasan $D$, Tengman $E$, Hager CK. Increased movement variability in one-leg hops about 20 years after treatment of anterior cruciate ligament injury. Clin Biomech (Bristol, Avon). 2018;53:37-45.

33. Zhang LQ, Shiavi RG, Limbird TJ, Minorik JM. Six degrees-of-freedom kinematics of $A C L$ deficient knees during locomotion-compensatory mechanism. Gait Posture. 2003;17:34-42.

34. Culvenor AG, Alexander BC, Clark RA, Collins NJ, Ageberg E, Morris HG, Whitehead TS, Crossley KM. Dynamic single-leg postural control is impaired bilaterally following anterior cruciate ligament reconstruction: Implications for reinjury risk. J Orthop Sports Phys Ther. 2016:46:357-64.

35. Kapreli E, Athanasopoulos S, Gliatis J, Papathanasiou M, Peeters R, Strimpakos N, Van Hecke P, Gouliamos A, Sunaert S. Anterior cruciate ligament deficiency causes brain plasticity: a functional MRI study. Am 」 Sports Med. 2009:37:2419-26.

36. Grooms DR, Page SJ, Nichols-Larsen DS, Chaudhari AM, White SE, Onate JA. Neuroplasticity associated with anterior cruciate ligament reconstruction. J Orthop Sports Phys Ther. 2017;47:180-9. 
37. Heijne A, Fleming BC, Renstrom PA, Peura GD, Beynnon BD, Werner S. Strain on the anterior cruciate ligament during closed kinetic chain exercises. Med Sci Sports Exerc. 2004;36:935-41.

38. van Melick N, Meddeler BM, Hoogeboom TJ, der Sanden MWG N-v,

REH $\vee$ C. How to determine leg dominance: The agreement between self-reported and observed performance in healthy adults. PLoS One. 2017;12:e0189876.

39. Benoit DL, Damsgaard M, Andersen MS. Surface marker cluster translation, rotation, scaling and deformation: Their contribution to soft tissue artefact and impact on knee joint kinematics. J Biomech. 2015;48:2124-9.

\section{Publisher's Note}

Springer Nature remains neutral with regard to jurisdictional claims in published maps and institutional affiliations.

Ready to submit your research? Choose BMC and benefit from:

- fast, convenient online submission

- thorough peer review by experienced researchers in your field

- rapid publication on acceptance

- support for research data, including large and complex data types

- gold Open Access which fosters wider collaboration and increased citations

- maximum visibility for your research: over $100 \mathrm{M}$ website views per year

At BMC, research is always in progress.

Learn more biomedcentral.com/submissions 